The Journal of Animal \& Plant Sciences, 31(2): 2021, Page: 468-479

ISSN (print): 1018-7081; ISSN (online): 2309-8694

\title{
ELUCIDATION OF POTATO TUBER RESPONSE TO FUSARIUM SOLANI VAR COERULEUM INFECTION TRANSCRIPTOME ANALYSIS
}

\author{
W. Wen-Zhong ${ }^{1,3 \downarrow}$, Z. Tong ${ }^{2}$, G. Rui ${ }^{4}$, C. Ling-Ling ${ }^{2}$, F. Peng ${ }^{2}$, Z. Zong-Bao ${ }^{2}$, Y. Shuai ${ }^{1}$, W. Qi ${ }^{1}$, G. Mei ${ }^{1}$, M. Fan- \\ Xiang ${ }^{1}$, H. Lin-Shuang ${ }^{1}$, M. Yan-Song ${ }^{5}$, W. Gui-Jiang ${ }^{6}$ and C. Ying ${ }^{2 *}$ \\ ${ }^{1}$ Potato Research Institute, Heilongjiang Academy of Agricultural Sciences, Harbin, 150086, China \\ ${ }^{2}$ College of Life Sciences, Northeast Agricultural University, Harbin, 150030, China \\ ${ }^{3}$ Heilongjiang Academy of Agricultural Sciences Postdoctoral Programme, Harbin, 150001, China \\ ${ }^{4}$ Center for Synthetic Biology, College of Chemical and Biological Engineering, Zhejiang University, Hangzhou 310027, \\ China; ${ }^{5}$ Beidahuang Kenfeng Seed Co. Ltd, Harbin, 150090, China; ${ }^{6}$ Heilongjiang Academy of Agricultural Sciences, \\ Harbin, 150001, China \\ *Corresponding author's e-mail: changying@neau.edu.cn \\ $\checkmark$ Authors contributed equally to this work.
}

\begin{abstract}
Dry rot in potatoes, caused by Fusarium spp. is a global critical post harvest fungal disease. To explore the response of Solanum tuberosum cv. Kexin No.13 to Fusarium solani var. coeruleum in potato tuber, we measured gene expression changes after $72 \mathrm{~h}$ pathogen inoculation by Illumina sequencing. The transcriptome analysis was used to identify candidate resistance genes and to dissect the early molecular processes. Of these, 4,262 differentially expressed genes (DEGs) were identified. The data were further confirmed by real-time PCR. The data indicated that F. solani var. coeruleum infection triggered DEGs were associated jasmonic acid (JA) and ethylene (ET) signaling pathways. Moreover, six kinds of transcription factors, three kinds of proteases and pathogenesis-related proteins (PRs) were also up-regulated in potatoes after pathogen inoculation. Overall, our findings give a broader view of the dynamic changes of the potato transcriptome triggered by $F$. solani var. coeruleum and highlights that the resistance response of potato is mainly signaled by JA and ET pathways, transcription factors, proteases, and PRs to enhance the host defence ability in potato tubers. All these could provide a genetic and molecular level foundation for the study on resistance to potato dry rot.
\end{abstract}

Key words: Fusarium solani var. coeruleum, molecular pathological response, Solanum tuberosum, transcriptome. https://doi.org/10.36899/JAPS.2021.2.0236

Published online October 03,2020

\section{INTRODUCTION}

With 17.58 million of tons produced, potatoes are the third most vital food crop in the world (http://www.fao.org/faostat; 2018). Potato dry rot, a soiland seed tuber-borne fungal disease (Peters et al., 2004) that commonly occurs after infection with Fusarium spp. and no potato cultivar could resistant to the entire Fusarium complex (Leach and Webb, 1981; Esfahani, 2005; Du et al., 2012; Chen et al., 2012; Yang et al., 2012; Shan et al., 2017), resulting in a 6 25\% loss in potato production (Stevenson et al., 2001). Although dry rot is the most economical post-harvest diseases, limited research at genetic and molecular level. It was reported that StCyp, StMBF1 and St-ACO3 genes were up regulated in $S$. tuberosum cv. Spunta after $F$. solani f. sp. eumartii inoculation (Godoy et al., 2000; Godoy et al., 2001; Zanetti et al., 2002; Blanco et al., 2006). The expression level of non-specific lipid transfer protein gene StLTPal showed fluctuation during F. culmorum infection and may participate in defense responses (Fu et al., 2016). RNA-Seq is an efficient, time saving and powerful approach for studying entire transcriptomes. Wheat - Fusarium interactions has been studied by using RNA-Seq (Erayman et al., 2015), but as far as know, this approach has never been used to study the defense responses in potato - Fusarium interaction.

Heilongjiang Province is the most important potato production areas in China. Potato dry rot caused by $F$. solani var. coeruleum is a serious threat in various locations of China and Heilongjiang Province (Ye and Wang, 1995; Min et al., 2010). Our research would perform to investigate the responses of selected potato cultivars to $F$. solani var. coeruleum. RNA-Seq analysis would provide us with more information of potato transcriptome in response to $F$. solani var. coeruleum and reveal molecular mechanisms in potato tubers.

\section{MATERIALS AND METHODS}

Plant and fungal material: Kexin No. 13 was used in this study which was a major potato variety in Heilongjiang Province. Virus-free potato tubers were harvested from a farm within the Virus-free Seedling 
Research Institute of Heilongjiang Academy of Agriculture Sciences (VSRI-HAAS), Harbin, China. Tubers were harvested on September 2015. Then packed in string bags and transported to the lab within $24 \mathrm{~h}$ of harvest. Tubers without damages or visible infections, and were stored at 5 to $8^{\circ} \mathrm{C}$.

F. solani var. coeruleum was obtained from VSRIHAAS and persevered at $25^{\circ} \mathrm{C}$. It was cultured on potato dextrose agar (PDA) one week for tested.

Fungal infection of potato tubers: Before treatment, the potato tubers were adapted at $25{ }^{\circ} \mathrm{C}$ for $24 \mathrm{~h}$ in the dark. Tubers were surface-sterilized by soaking in a $5 \% \mathrm{NaOCl}$ $5 \mathrm{~min}$, then flushed by distilled water, nature drying and inoculated using a modified as Radtke and Escande (1973) mentioned. First, make a hole $5 \mathrm{~mm}$ in diameter and $3 \mathrm{~mm}$ deep on the surface of tuber. Then the treatment tubers were inoculated by a mycelial disk ( 5 $\mathrm{mm}$ diameter) of $F$. solani var. coeruleum. Inoculation treatments with a PDA disk was control. All treated potato tubers were placed to plastic boxes lined with sterile moistened tissue, with incubation at $25{ }^{\circ} \mathrm{C}$ and 80 $90 \%$ humidity. Each treatment contained three replicates, and the experiment was repeated twice.

RNA Extraction, cDNA Library Construction and Sequencing: Potato samples from without (CK) and with inoculation treatments $(\mathrm{T})$ were collected after $72 \mathrm{~h}$ inoculation. The potato tubers from CK extracted RNA individually, and then the same was done with the three tubers with $T$. The two replicated experiments samples of $\mathrm{CK}$ and $\mathrm{T}$ experimental samples were used to prepare each RNA with the same method as above. To avoid individual differences in individual potatoes, six samples from different potato tubers of $\mathrm{CK}$ and $\mathrm{T}$ experimental samples were used to extract each RNA sample as the two experimental repeats.

RNA purified and extracted by OminiPlant RNA Kit (DNase I) (CW2598M, CWBIO, Beijing, China) in accordance with the manufacturer manual. The quality and quantity of the total RNA was validated using the 2000C spectrophotometer (Nanodrop, Wilmington, DE), Qubit 2.0 Fluorometer (ThermoFisher, Waltham, MA) and 2100 Bioanalyzer (Agilent Technologies, Santa Clara, CA), respectively. Two mixtures of same amounts of high quality RNA samples were ready to generate cDNA libraries. The cDNA libraries were sequenced on the Illumina $\mathrm{HiSeq}^{\mathrm{TM}} 2000$ platform at Biomarker Technologies Corporation, Beijing, China.

The RNA was enriched using Oligo (dT) magnetic beads (Illumina Inc., San Diego, CA, USA), and fragmented using fragmentation buffer (Illumina). The cDNA was synthesized using a primer with six random hexamers, then purification using AMPure XP beads purification kit (Beckman Coulter, Inc. CA, USA), and then the adaptors ligation, AGE purification and PCR amplification. The PCR products of libraries were subjected to Illumina HiSeq ${ }^{\mathrm{TM}} 2000$ (Illumina Inc., San Diego, CA, USA).

Transcriptome Assembly and Analysis: There are two cDNA libraries were sequenced $-\mathrm{CK}$ and $\mathrm{T}$. The clean reads were received, after filtering the low-quality sequences, adaptor-pollution and high content of unknown base reads. After trimming and quality evaluation, for further analysis we used bowtie2 with tophat2 (http://ccb.jhu.edu/software/tophat/index.shtml) to map the useful reads for $S$. tuberosum genes (http://solanaceae.plantbiology.msu.edu/pgsc_download.s $\mathrm{htmL}$ ) and the reference genomes and to obtain the mapped reads.

Then using BLAST (Altschul et al., 1997), all the unigenes in $S$. tuberosum transcriptome obtained here were combined with multifarious protein databases in the following order: NCBI, non-redundant protein $(\mathrm{Nr})$ database, Swiss-Prot, Kyoto Encyclopedia of Genes and Genomes (KEGG), Cluster of Orthologous Groups of proteins (COG), Gene Ontology (GO).

As a sign, FPKM is used to calculate the expression of transcriptional or gene (Florea et al., 2013).

Differential Expression Analysis of Genes: In addition, DEGs were analyzed using the EBSeq package (Leng et al., 2013) with False Discovery Rate of 0.05. DEGs of transcripts are an independent statistical hypothesis for a large number of gene expression values. BenjaminiHochberg correction methods were used on the significance of original hypothesis test to get the $p$ value ( $\mathrm{p}$ - value) for correction.

Validation of RNA-Seq Data Using Real-Time PCR: Nine genes were selected for validation and EF1- $\alpha$ served as an internal gene control (Table 1). The gene specific primers were diluted to $25 \mathrm{mM}$. The RNA samples were obtained using the same treatment method as RNA-seq. After reverse transcription, the real-time PCR was performed in Lightcycler 480 (Roche, USA) using UltraSYBR Mixture (CWBIO, China) followed the protocol. The following program: initial denaturation at $95^{\circ} \mathrm{C}$ for $10 \mathrm{~min}$, then 40 cycles of $95^{\circ} \mathrm{C}$ for $15 \mathrm{~s}, 60^{\circ} \mathrm{C}$ for $1 \mathrm{~min}, 95{ }^{\circ} \mathrm{C}$ for $15 \mathrm{~s}$ and $72{ }^{\circ} \mathrm{C}$ for $15 \mathrm{~s}$. The experiments were each repeated three times. Valuation of the relative amounts of amplification products as Kidd et al. (2001) described.

\section{RESULTS AND DISCUSSION}

Overall of RNA-Seq and reads assembly: RNA-Seq analysis was carried out on total RNA samples from mock (CK) and F. solani var. coeruleum-inoculated sample (T), in order to identify DEGs putatively and pathways involved in the resistance response. The RNA integrity number (RIN) of CK and T were 6.6 and 7.3, OD260/280 were 2.13 and 2.13, OD260/230 were 1.98 
and 2.3, respectively. Sequencing generated 16,988,401 (CK) and 20,244,939 (T) clean reads that were representative of $4.27 \mathrm{~Gb}$ and $5.09 \mathrm{~Gb}$ of sequence data, respectively (Table 2). Sequences from both of these two libraries exhibited overall GC content of $\sim 50 \%$ with Phred quality scores (Q30) above $89.50 \%$, demonstrating that the sequencing data were accurate and sufficient for further analysis. The length of the sequence reads was 125 bp (Additional file 1: Fig. S1). The length distributions of total unigenes (Additional file 2: Fig. S2) had similar patterns between the libraries, suggesting that there was no bias in the library construction.

Further analysis using the EBSeq package revealed that 4,262 DEGs were expressed with2-fold difference or greater difference in T from CK. Amongst these DEGs, 2,662 were up-regulated and 1,600 were down-regulated. Overall, 3,542 DEGs were allocated to gene ontology (GO) classes (Fig. 1), which were further categorised using $53 \mathrm{GO}$-assigned general parent terms.

After comparing sequences to databases of clusters of orthologous groups (COGs), 1,743 DEGs were allocated to $23 \mathrm{COG}$ classifications (Fig. 2). However, no DEGs were allocated to "nuclear structure" or "cell motility". It was noteworthy that "replication, recombination and repair" (228), "secondary metabolites biosynthesis, transport and catabolism" (191) and "carbohydrate transport and metabolism" (181) were also frequent matches. Therefore, these results should stimulate future research to elucidate the interrelationships and functions of genes induced by $F$. solani var. coeruleum infection.

KEGG pathway enrichment analysis determines whether DEGs are significantly represented within a particular pathway. The results (Fig. 3) demonstrate that 1,342 DEGs from the infected potato library were assigned to 114 KEGG pathways. "Metabolism" (332), "genetic information processing" (66) and "environmental information processing" (33) were the most common annotations observed for the 20 KEGG pathways most frequently assigned (Fig. 4). F. solani var. coeruleum is a serious threat fungus pathogen for potato production and quality and other crops. A lack of available genetic or genomic information for potatoes during infection by $F$. solani var. coeruleum has hindered identification of potato dry rot resistance related mechanism. Genome-wide gene expression profiles can help elucidate the molecular mechanisms underlying host responses to pathogen infections. Now, potato dry rot caused by Fusarium spp. is not clear. Thus, analysis of this research could help to elucidate potato tuber transcriptional responses during the host fungal interaction and have great significance for further study. In this study, transcriptome changes were analysed using Illumina sequencing in order to investigate the defence mechanisms of potato (Kexin No. 13) to F. solani var. coeruleum. The results focused mainly on pathogen- inoculated transcriptome changes in potato, the resulting data add to the available genetic resources for exploring potato developmental biology and defence mechanisms in response to pathogen.

Gene associated with plant hormones: Plant hormones are organic substances, which regulate growth and produced in response to environmental factors. Many hormones, such as JA, methyl jasmonate (MeJA), salicylic acid (SA), ET and brassinolide (BR) are signal molecules contained plant defence against pathogens. The key enzymes in JA biosynthesis are allene oxide cyclase (AOC), allene oxide synthase (AOS), lipoxygenase (LOX) and 12-oxophytodienoate reductase (OPR). As avital regulator of JA signaling, jasmonate ZIM-domain (JAZ) protein controls DNA-transcription factor activity when no JA is present in a plant (Sun et al., 2014). We found DEGs encode OPR, AOS and JAZ (Table 3), and did not detect genes encoding AOC and LOX which probably because they were not expressed at the start of transcription or are not present in potato. ET is also an important signal for the induction of wound and insect attack responses. The modulation of expression of ET biosynthesis is controlled by 1-aminocyclopropane-1carboxylate oxidase (ACC oxidase) and 1aminocyclopropane-1-carboxylate synthase (ACC synthase). After inoculation, ten DEGs encoding ACC oxidase and three DEGs identified as ACC synthase and were all up-regulated (Table 3). This result indicated that only ACC synthase as an indication for ET biosynthesis after F. solani var. coeruleum inoculation.

In response to stresses and adjust physiological reactions, plant hormones are generated. JA biosynthesis pathways are connected with the oxylipin and octadecanoid synthetic pathway in which AOS and OPR are important synthetases that participate in positive regulatory control mechanisms (Sasaki et al., 2001). JAZ proteins, which are found only in angiosperm and gymnosperm, are up-regulated soon after plants are wounded or receive JA treatment. They play a central inhibitory mission in JA signaling and have the induction for early gene transcription response (Sun et al., 2014; Chini et al., 2007). One JAZ gene isolated from grape could improve resistance and enhance defense responses through JA signaling pathway to fungal pathogens in Arabidopsis thaliana (Zhang et al., 2019). ACC oxidase and ACC synthase are rate-limiting enzymes in ET biosynthesis.

As shown in Table 3, after $F$. solani var. coeruleum inoculation, AOS, OPR and JAZ genes were upregulated, with the most pronounced effect observed for ACC synthase and ACC oxidase genes. These results suggest that $F$. solani var. coeruleum could accelerate JA and ET biosynthesis.

Genes related to transcription factors: Plant resistance responses are composed of many defense genes and 
transcription factors. Many transcription factors participate in plant resistance, including MYB, WRKY, ERF and MYC, as do proteins with bHLH and WD40 structural motifs. Resistance-associated transcription factors can activate plant defence responses or may be involved directly in various defence signaling pathways. Based on our transcription data, a total of 76 DEGs were identified as WRKY (38 DEGs), MYB (12 DEGs), MYC (3 DEGs), ERF (21 DEGs), bHLH (1 DEG) and WD40 (1 DEG). Amongst these 76 DEGs, 60 DEGs were upregulated (Table 3 ). These showed that after F. solani var. coeruleum inoculation, genes which encode transcription factors have effective on potato resistance to the pathogen studied here.

WRKY is a superfamily of transcription factors particular to plants and in higher plants. Over expression of the Malus domestica MdWRKY100 gene could stimulate resistance to Colletotrichum gloeosporioides and has positive regulation in apple (Zhang et al., 2020). Additionally, MYC2 (a basic bHLH transcription factor), ERF1, ERF2, ERF4 (AP2/ERF transcription factors) and various affiliates of WRKY transcription factor family participate in JA-induced signal pathway and play vital parts in JA stimulation (Lorenzo et al., 2004). MYC2 can directly combine to the JAZ promoter T/G-box domain and regulates the expression of JA response genes (Chini, 2009). The AP2/ERF transcription factor family shares a highly conserved DNA-binding domain with an ERF domain structure; its expression levels are adjusted to answer environmental stresses. Another defence response strategy utilizes ERF proteins. ERFs are involved in pathogen defence and signal transduction pathways and are cross-talk factors in stress signal pathways (Zhang et al., 2012). ERF is located downstream of the ethylene signaling pathway, and involved in the ethylene signaling pathway resistance to pathogens. As reported, MdERF11 in apples plays a significant role in activating phytoimmunity response to Botryosphaeria dothidea (Wang et al., 2020). As yet another defence in plants, MYB family members are important elements in adjustment and controlling for development, metabolism and reactions to pathogens and they all contain common MYB structural domains. MYB transcription factors play a crucial part in plant resistance. OsMYC2 is a JAZ transcription factor, has great affection the resistance answer to Xanthomonas oryzae in rice (Uji et al., 2016). CaPHL8 is an MYB transcription factor and has positive regulator of pepper defense against Ralstonia solanacerum inoculation (Noman et al., 2019).

In this research, most transcription factors were significantly up regulated and we assumed that these genes play important roles in resistance to $F$. solani var. coeruleum infection. Mover, WRKY and ERF transcription factors also play vital roles in JA biosynthesis and signaling pathways after $F$. solani var. coeruleum treatment.
Plant proteolytic function in defence responses: Plant proteases widely existing in plants, they not only degrade nonfunctional proteins into amino acids but also play key roles for regulation. After pathogens infection, many plants enhance resistance by production proteases. From our transcription results we could found three kinds proteases and 18 DEGs were identified, including serine protease (12 DEGs), aspartic proteases (APs) (5 DEGs) and metalloproteases (1 DEG). Serine protease is the largest protease family in plants and divided into 14 families. In our results, 7 DEGs were identified as subtilisin-like protease, 2 DEGs were identified as protease Do-like, 1 DEG was identified as rhomboid protease and 1 DEG was identified as Clp protease. Five DEGs encoding APs, only one DEG encoding metalloproteases.

Serine proteases are the largest proteolytic enzymes in plant, and subtilisin-like protease and serine carboxypeptidase protease-like proteins are the biggest components of serine protease. Subtilisin-like proteases may act as convertases in plant. Subtilases P69B and $\mathrm{P} 69 \mathrm{C}$ in tomato were induced by citrus exocortis viroid and following pathogen infection and SA application (Tornero et al., 1996). We observed that after $F$. solani var. coeruleum inoculation there were seven DEGs were identified as subtilisin-like protease and five were upregulated. These data show that after $F$. solani var. coeruleum treatment serine proteases, especially for subtilisin-like protease, may play important roles in response to pathogen. APs are important proteolytic enzymes which are widely distributed in mammals, plants, bacteria and viruses. They also participate in the process of plant disease resistance, art resistance and leaf senescence. After Phytophthora infestans inoculation, APs were induced from different potato cultivars (Guevara et al., 2002). Transformed S. tuberosum aspartic proteases (StAP-PSI) to A. thaliana, and it remarkable enhances resistance against $B$. cinerea infection by increased content of StAP-PSI and expression level of induced defense genes. This constitutive expression of StAP-PSI also regulates JA signaling pathway (Frey et al., 2018). In this study, the results showed that the genes identified as AP were up-regulated, and some genes identified as JA signaling pathway were almost also upregulated. This paper shows that after $F$. solani var. coeruleum treatment, potato maybe active AP activity and through JA signaling pathway. Besides the characteristics of general protease, the most important feature of metalloproteinase is that the active center depends on some metal ions and can be strongly inhibited by metal complexing agent. Filamentation temperature sensitive $\mathrm{H}$ (FtsH) belongs to metalloprotease and DS9 gene encodes a chloroplastic homolog of bacterial FtsH protein. After TMV infection, DS9 protein reduced and indicated that DS9 is related to resistance and accelerates the hypersensitive reaction (Seo et al., 2000). This paper we 
found one DEG encoding FtsH, after $F$. solani var. coeruleum treatment FtsH was also down-regulated.

Plant PR genes in defence responses: A lot of research showed that after pathogen infection some specific genes would express and affect the pathogenic ability. Plant protects itself through the synthesis of various PRs which have antimicrobial properties. PR proteins are induced by pathogens and abitotic stresses, and important components for defence system. The genes encode PR proteins expressed increased after induction. Based on PR proteins' structural and functional properties, all together 17 disparate families have been found. 1,3-beta-glucanase and chitinase are important PR proteins, and establish a close relationship for resistance. 1,3-beta-glucan and chitin are vital structural composition of fungal cell walls. Previous research showed that overexpression of PnGlu1 gene, which was a beta-1,3-glucanase gene and characterized from Panax notoginseng, could strong resistance to $F$. solani in tobacco (Taif et al., 2020). By transformation alfalfa $\beta$-1,3-glucanase (AGLU1) and rice chitinase (RCH10) genes, oil palm were completely tolerant to basal stem rot (BSR) disease (Hanin et al.,
2020)

In this study, we found 12 DEGs were identified as chitinase and 5 DEGs were identified as 1,3-betaglucanase. All genes exhibited the same expression pattern and all up-regulated I, potatoes would synthetic $\mathrm{PR}$ proteins to increase the resistance.

Confirmation of expression patterns using real-time PCR: In order to validate our DEGs data, we conducted real-time PCR analysis to confirm the validity of expression changes observed without or with $F$. solani var. coeruleum inoculation. Some genes related to transcription factors, proteolytic activity, plant defence, and PR-protein, were tested. For T sequences, the values are presented as the fold change in gene expression (after normalization to the reference gene, actin) relative to $\mathrm{CK}$ samples. Real-time PCR results (Fig. 5) for these DEGs were almost identical to the Illumina sequencing results. However, any discrepancies in expression levels between these two data sets may be due to the greater sensitivity of Illumina sequencing vs. that of real-time PCR.

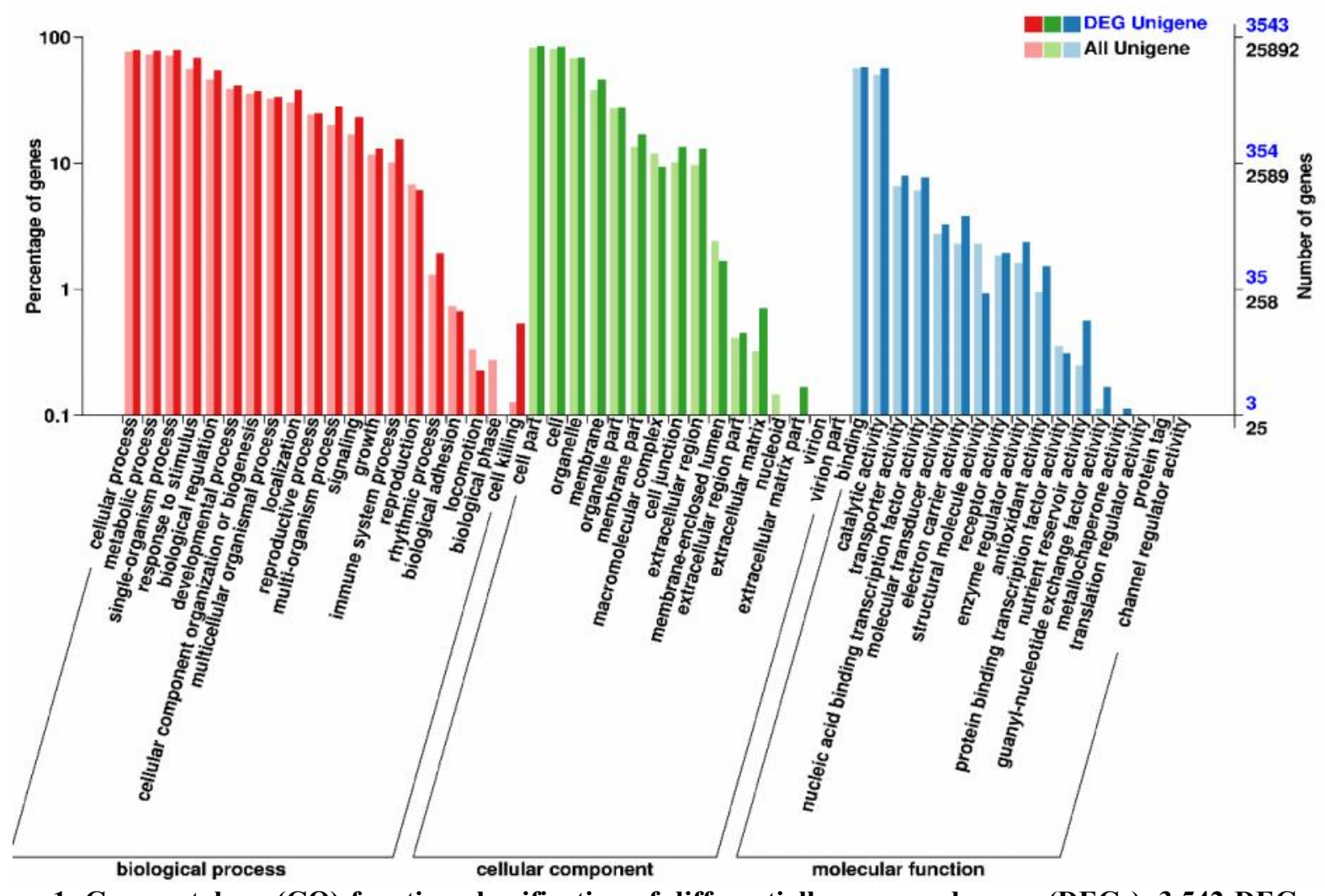

Figure 1. Gene ontology (GO) function classification of differentially expressed genes (DEGs). 3,542 DEGs were categorized into three main categories (biological process, cellular component, and molecular function) and 53 functional groups.

The $\mathrm{x}$-axis shows the functional groups. The right $y$-axis shows the number of genes in a category. The left y-axis shows the percentage of a specific category of genes in that main category. 


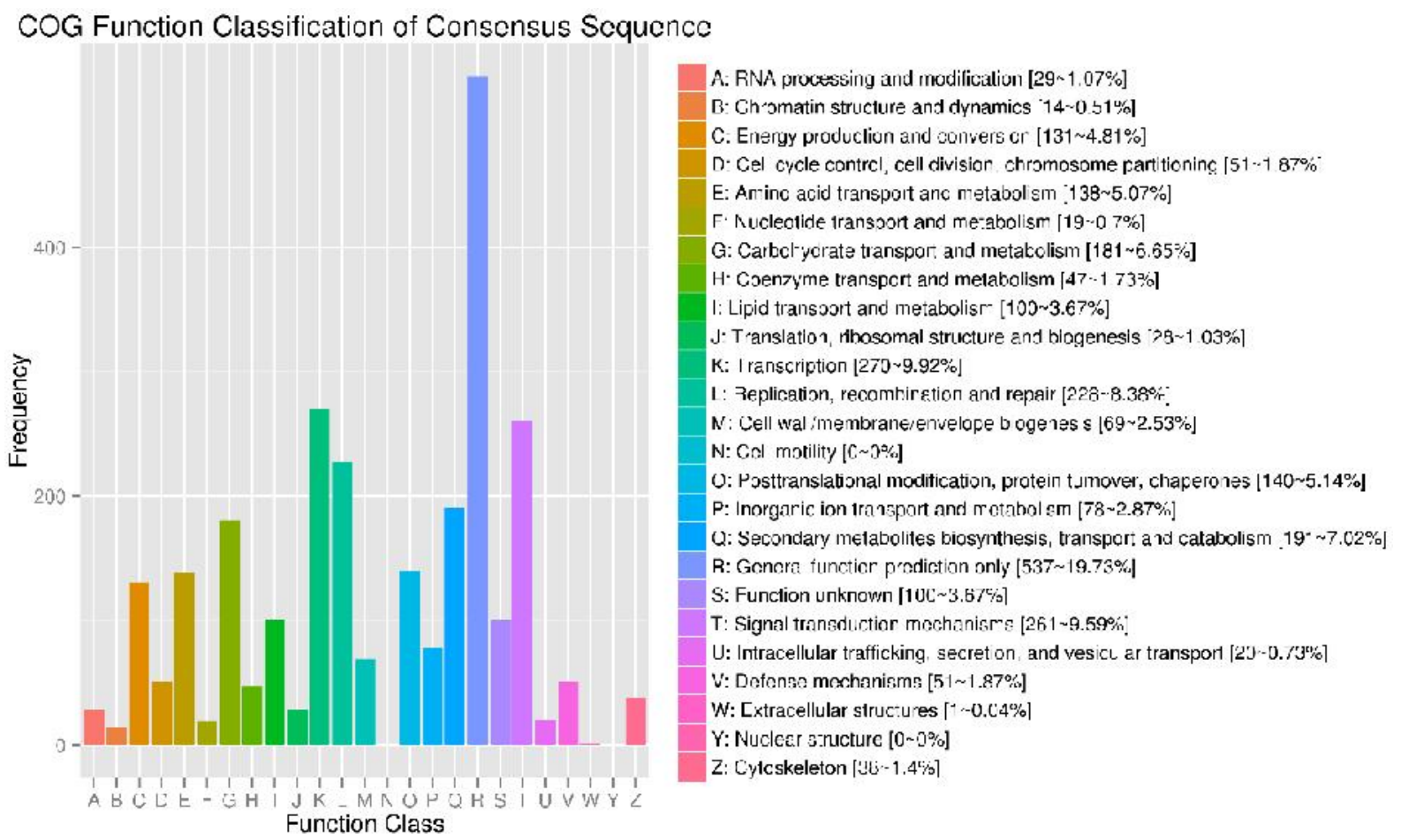

Figure 2. COG function classification of DEGs. 1,743 DEGs were allocated to 23 COG classifications. The $x$-axis shows the function class. The y-axis shows the frequency of DEGs in a category.

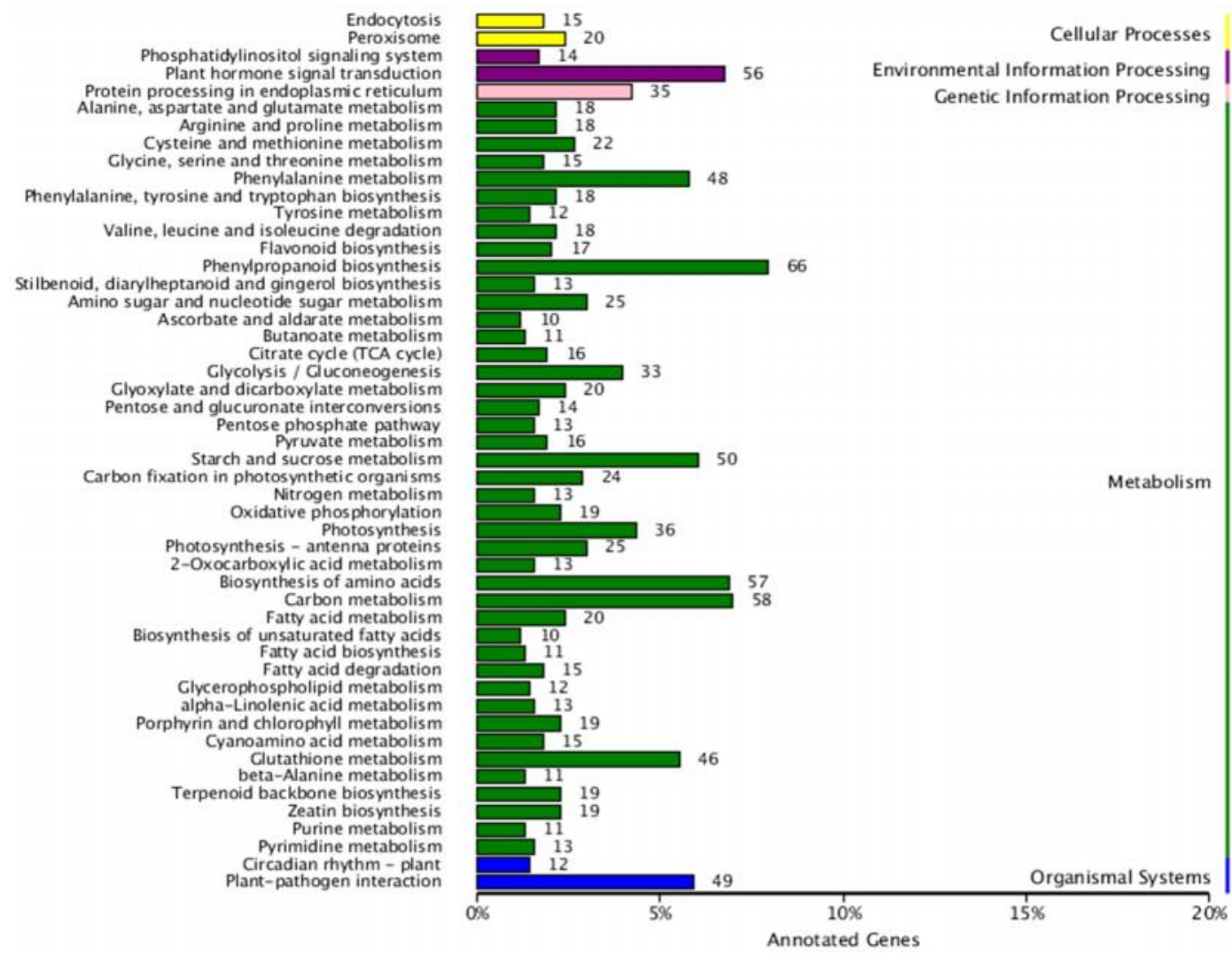

Figure 3. KEGG pathways enrichment analysis of DEGs. 1,342 DEGs were allocated to 114 KEGG pathways. The $\mathrm{x}$-axis shows the number of annotated genes. The $\mathrm{y}$-axis shows the KEGG pathway category. 


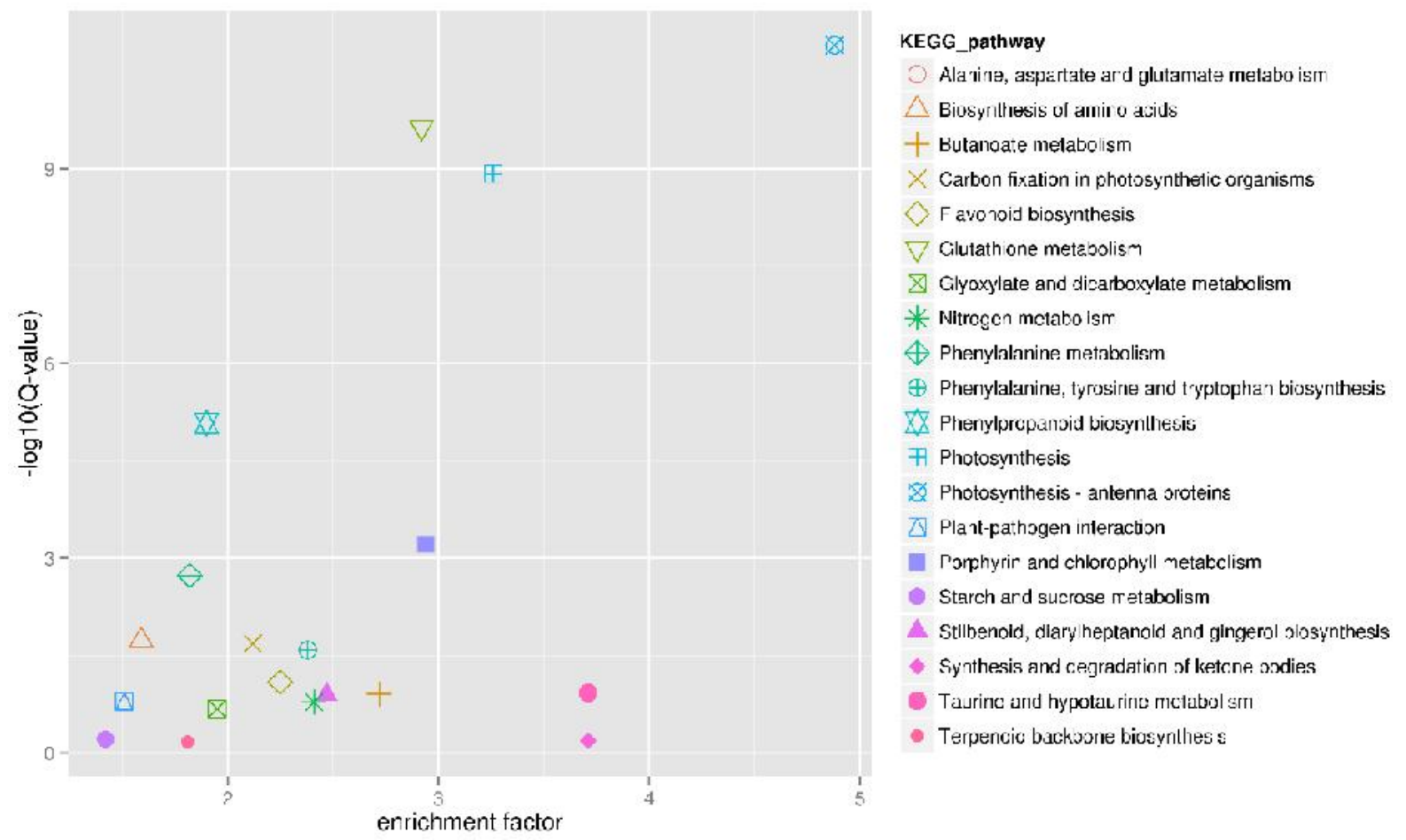

Figure 4. Important KEGG pathways infected with Fusarium solani var. coeruleum.

The $\mathrm{x}$-axis shows the enrichment factors. The $\mathrm{y}$ axis shows the significance of enrichment of genes. (Qvalue is the $\mathrm{p}$-value after multiple hypotheses testing. The larger value of y-axis, the more reliable of DEG enrichment in the KEGG pathway).
Q Real-time PCR

Illumina sequencing

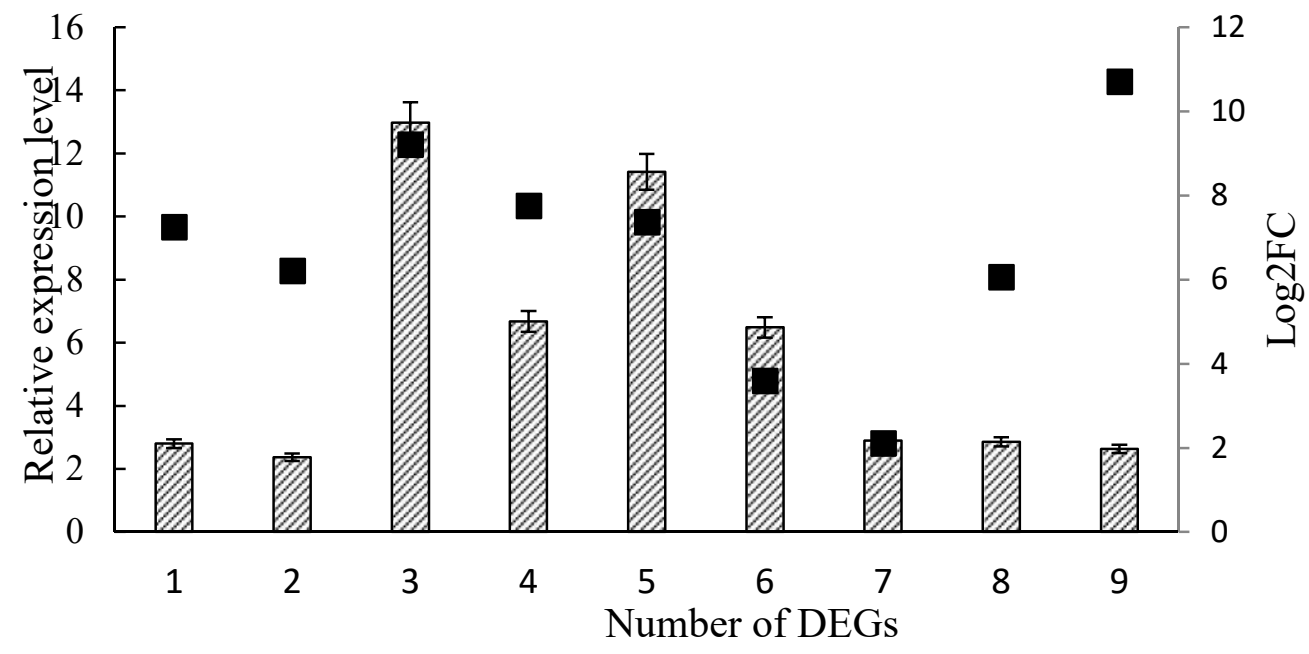

Figure 5. Real-time PCR analysis for nine up-regulated DEGs with Fusarium solani var. coeruleum (T) or without pathogen-inoculated $(\mathrm{CK})$ of potato samples.

Validation of the relative expression was $\mathrm{T}$ over CK. The left and right vertical ordinates indicate selected gene expression determined by real-time PCR and RNA sequencing, respectively. Real-time PCR was carried out 
on three independent biological replicates each containing three technical replicates. Relative expression level, detection of real-time PCR; Log2 FC, fold change of the DEGs in the transcriptome.
1. ACC oxidase gene; 2. ACC oxidase gene; 3. WRKY gene; 4. ERF gene; 5. MYB gene; 6. WD40 gene; 7. subtilisin-like protease gene; 8. Serine protease inhibitor gene; 9. Chitinase gene.

Table 1. Primers used in this research

\begin{tabular}{|c|c|c|c|c|}
\hline $\begin{array}{c}\text { No } \\
\text {. }\end{array}$ & Gene ID & $\begin{array}{c}\mathrm{Nr} \\
\text { annotation }\end{array}$ & Forward primers (5' to 3') & Reverse primers (5' to 3') \\
\hline 1 & PGSC0003DMG400025439 & $\begin{array}{c}\text { ACC } \\
\text { oxidase }\end{array}$ & TGGGAAGTCTAAACTCTATACGG & $\begin{array}{c}\text { ATTAATTTGAGGCAGATAACAA } \\
\text { CC }\end{array}$ \\
\hline 2 & PGSC0003DMG401025438 & $\begin{array}{l}\text { ACC } \\
\text { oxidase }\end{array}$ & CAAATGGCTGAAATTGAGTTGTG & $\begin{array}{c}\text { ATATCTCTTGTGTGACCTGATG } \\
\text { G186 }\end{array}$ \\
\hline 3 & PGSC0003DMG400011633 & WRKY & GTCAACCATCGCAATATGTTAGAG & $\begin{array}{c}\text { AGTAATGTGTCCATCCAAGTTC } \\
\text { C }\end{array}$ \\
\hline 4 & PGSC0003DMG400014594 & ERF & ATCСТCAAACACTACTACAACAAC & AGCCAAACCCTAACTCCATTAC \\
\hline 5 & PGSC0003DMG400000349 & MYB & AATTAAGGTGGTCGGTGATAGC & $\begin{array}{c}\text { TTGTTGGTGAAATTGTAGCCTA } \\
\text { TG }\end{array}$ \\
\hline 6 & PGSC0003DMG400026477 & $\begin{array}{l}\text { WD40 } \\
\text { subtilisin- }\end{array}$ & TTCATCCTAATCCTTCTGCTTCTC & CGGCTCCACTTCATTCCAATC \\
\hline 7 & PGSC0003DMG400006841 & $\begin{array}{l}\text { like } \\
\text { protease } \\
\text { serine }\end{array}$ & GCTTCAGTGCTGTTTTGT & GTGGGTAGTATGAGGTTCC \\
\hline 8 & PGSC0003DMG400015267 & $\begin{array}{l}\text { protease } \\
\text { inhibitor }\end{array}$ & ATTGGAGACGGGAGGAAC & GATGGACAAACAAAAGGAC \\
\hline 9 & $\begin{array}{l}\text { PGSC0003DMG400001528 } \\
\text { Internal reference }\end{array}$ & $\begin{array}{l}\text { chitinase } \\
\text { EF } 1-\alpha\end{array}$ & $\begin{array}{c}\text { GAATGTGGGATGGGTCAG } \\
\text { TGCCCAGAAGAAGGGAAAGTGA }\end{array}$ & $\begin{array}{c}\text { GGGCGAAGTTCCTTTGAT } \\
\text { AATCATGCTCGCCACCGCCTAT }\end{array}$ \\
\hline
\end{tabular}

Table 2. Summary statistics for potato genes infected without or with Fusarium solani var. coeruleum based on the RNA-Seq data

\begin{tabular}{ccc}
\hline & Control (CK) & F. solani var. coeruleum-inoculated (T) \\
\hline RNA integrity numbers (RIN) & 6.6 & 7.3 \\
Clean reads & $16,988,401$ & $20,244,939$ \\
Total reads & $33,976,802$ & $40,489,878$ \\
Sequence data & $4.27 \mathrm{~Gb}$ & $5.09 \mathrm{~Gb}$ \\
GC Content & $43.81 \%$ & $43.98 \%$ \\
\% $\geq$ Q30 & $89.83 \%$ & $89.50 \%$ \\
Mapped reads & $24,284,350$ & $27,571,251$ \\
& $(71.47 \%)$ & $(68.09 \%)$ \\
Unique Mapped Reads & $22,165,699$ & $25,030,682$ \\
& $(65.24 \%)$ & $(61.82 \%)$ \\
Multiple Map Reads & $2,118,651$ & $2,540,569$ \\
\end{tabular}

Table 3. Summary of differentially expressed genes (DEGs) annotated as resistance-related genes in potato after Fusarium solani var. coeruleum inoculation

\begin{tabular}{|c|c|c|c|c|}
\hline \multirow{8}{*}{ Transcription factors } & ACC oxidase & 5 & 5 & 10 \\
\hline & ACC synthase & 3 & 0 & 3 \\
\hline & MYC & 1 & 2 & 3 \\
\hline & WRKY & 35 & 3 & 38 \\
\hline & ERF & 19 & 2 & 21 \\
\hline & MYB & 4 & 8 & 12 \\
\hline & basic helix-loop-helix (bHLH) & 0 & 1 & 1 \\
\hline & WD40 & 1 & 0 & 1 \\
\hline Proteases and protease inhibitor & Cysteine protease inhibitor & 0 & 2 & 2 \\
\hline
\end{tabular}




\begin{tabular}{ccccc}
\hline & Serine protease inhibitor & 1 & 2 & 3 \\
& Aspartic protease inhibitor & 0 & 1 & 1 \\
& Serine proteases & 6 & 6 & 12 \\
& Aspartic proteases & 3 & 2 & 5 \\
& Metalloproteases & 0 & 1 & 1 \\
Anti-oxidation & Peroxidase (POD) & 15 & 1 & 16 \\
\hline
\end{tabular}

\section{Additional files}

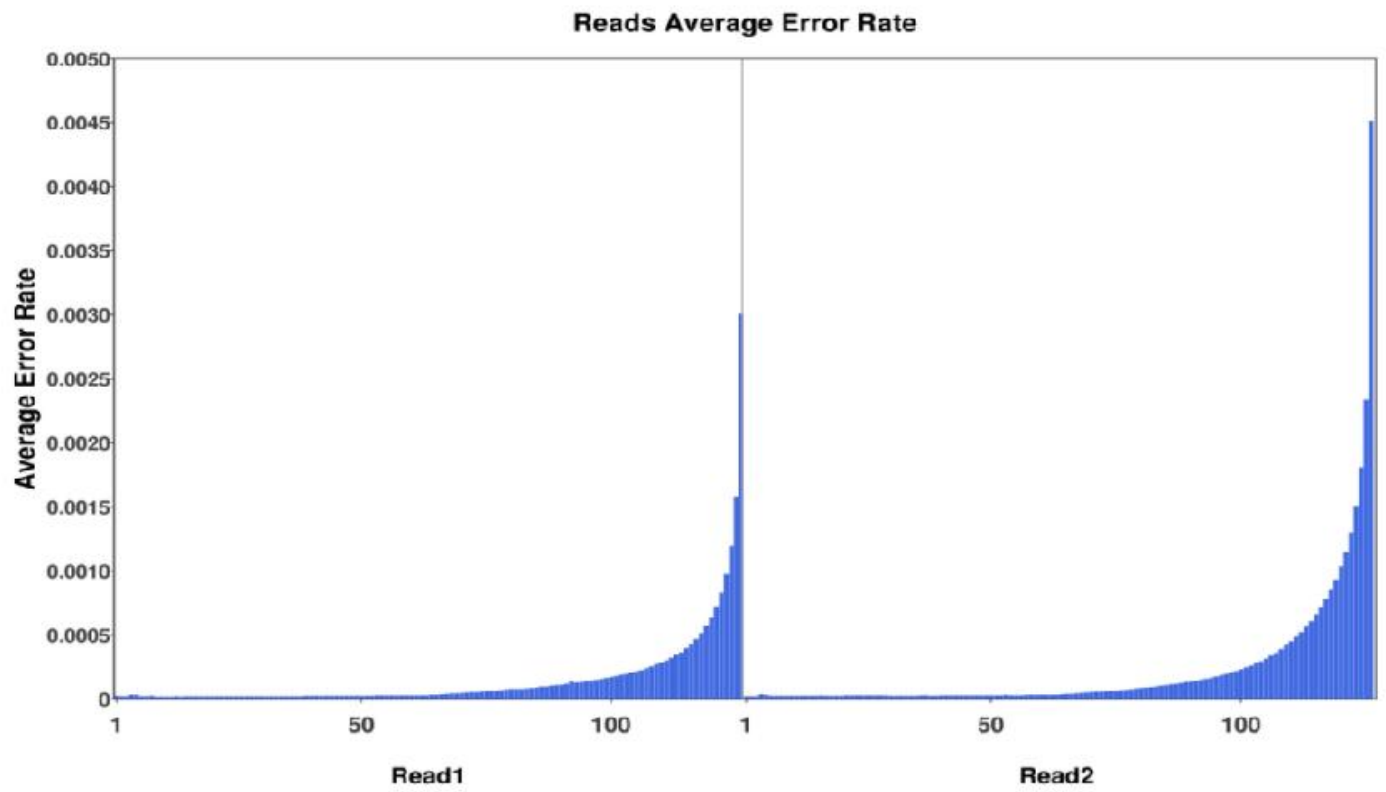

A. Reads average error rate distribution of unigenes in the assembled transcriptomes of potato genes inoculated without pathogen (CK).

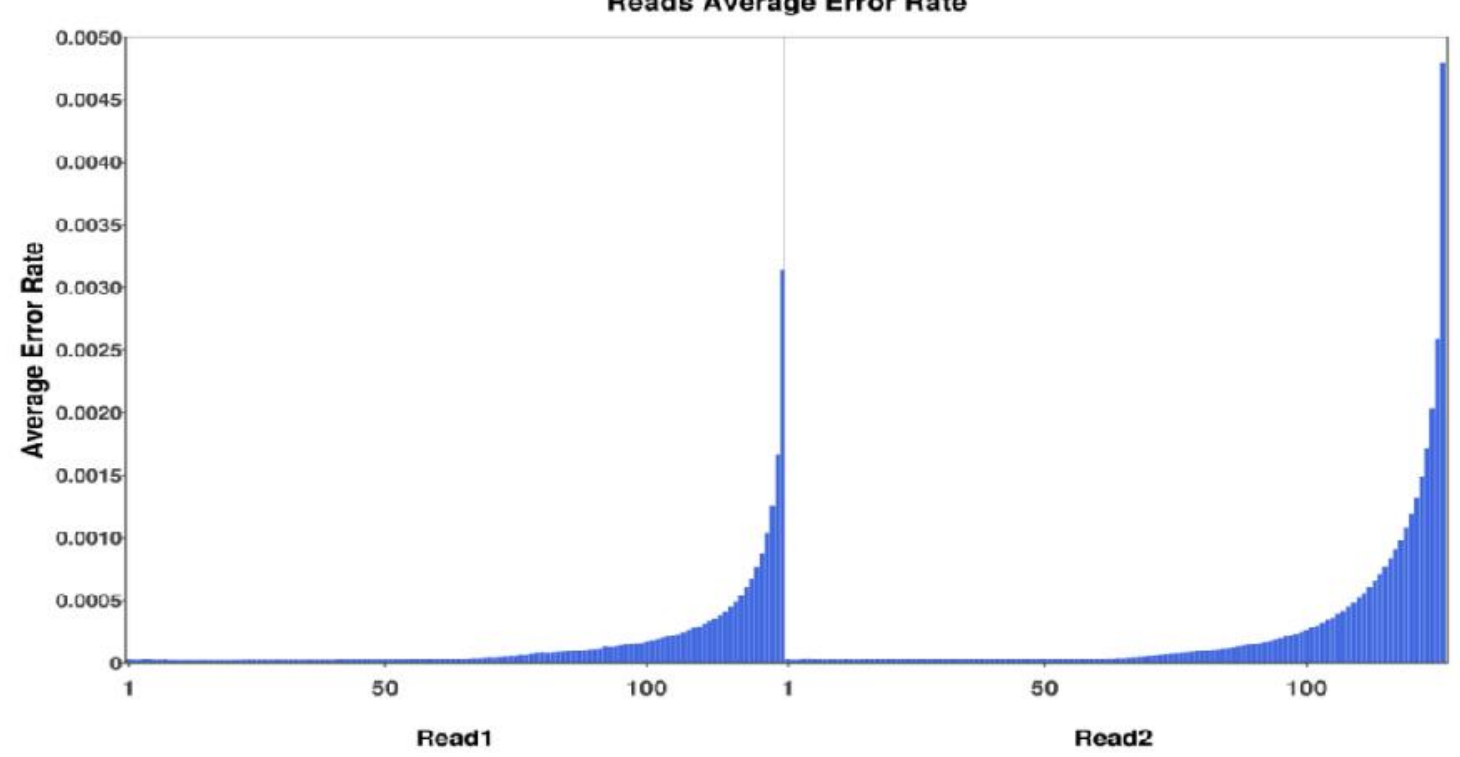

B. Reads average error rate distribution of unigenes in the assembled transcriptomes of potato genes inoculated with Fusarium solani var. coeruleum (T).

Figure S1. Reads average error rate distribution of unigenes in the assembled transcriptomes of potato were inoculated without (CK) or with Fusarium solani var. coeruleum (T).

The $\mathrm{x}$-axis shows the read base position of unigenes. The y-axis shows the average of error rate in our library. 


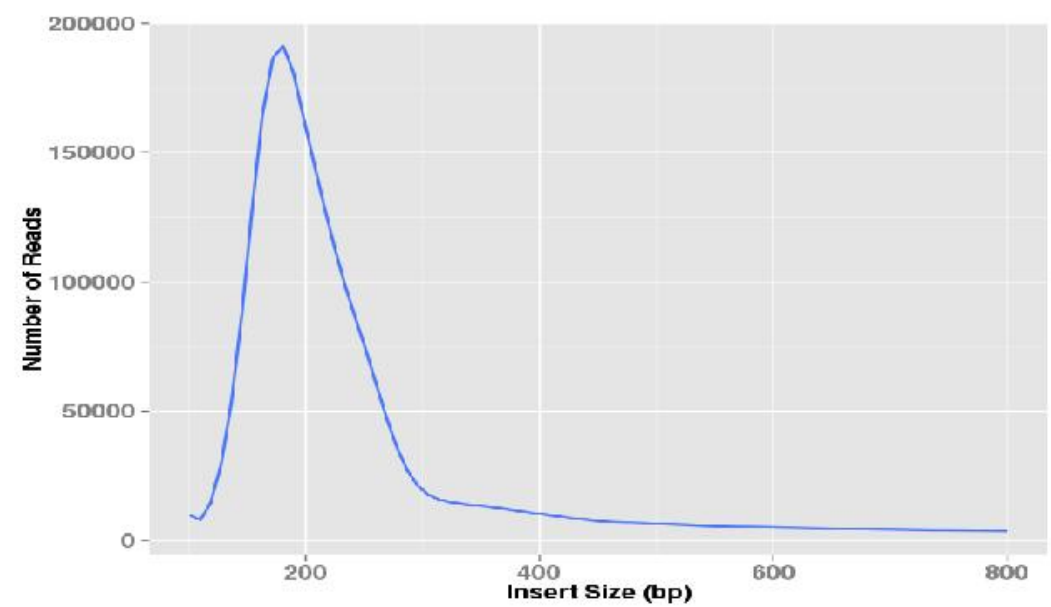

A. Length distribution of unigenes in the assembled transcriptomes of potato genes inoculated without pathogen (CK).

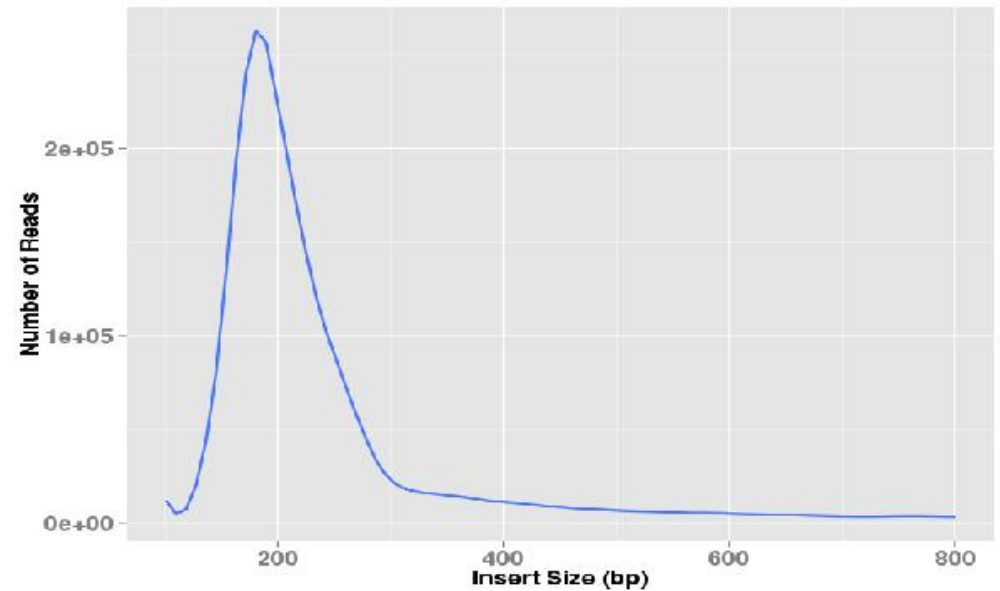

B. Length distribution of unigenes in the assembled transcriptomes of potato genes inoculated with Fusarium solani var. coeruleum (T).

Figure S2. Length distribution of unigenes in the assembled transcriptomes of potato genes were inoculated without (CK) or with Fusarium solani var. coeruleum (T). The $\mathrm{x}$-axis shows the lengths of unigenes and the $y$-axis shows the number of unigenes calculated in our library.

Conclusions: The transcriptome analysis in potato $-F$. solani var. coeruleum were characterized by RNA-Seq, the results suggested that after infection, various DEGs associated to signalling pathways, transcription factors, proteinase, and defence response related genes were differentially expressed. The DEGs up-regulated involved in JA and ET signaling pathways to accelerate resistance. At the same time, six kinds of transcription factors have effective on potato resistance, WRKY and ERF transcription factors play vital roles in JA biosynthesis and signaling pathways. Three kinds of proteases and two kinds of PRs were related to the defense response in inoculated potatoes. Overall, our findings reveal that the response to $F$. solani var. coeruleum inoculation are related with a complex resistance network. Further researches will be concentrated on validation and mapping of the selected DEGs, which could provide a genetic and molecular level foundation for developing potato resistant to dry rot caused by $F$. solani var. coeruleum.

Acknowledgements: This research was supported by National Key RandD Program of China (2017YFE0115700) and Heilongjiang Postdoctoral Financial Assistance (LBH-Z18267).

\section{REFERENCES}

Altschul, S.F., T.L. Madden, A.A. Schäffer, J. Zhang, Z. Zhang, W. Miller, and D.J. Lipman (1997). Gapped BLAST and PSI-BLAST: a new generation of protein database search programs. Nucleic Acids Research. 25(17): 3389-3402.

Blanco, F.A., M.E. Zanetti, C.A. Casalongué , and G.R. 
Daleo (2006). Molecular characterization of a potato MAP kinase transcriptionally regulated by multiple environmental stresses. Plant Physiology and Biochemistry. 44(5): 315-322.

Chen, H., J. Li, Z. Chai, C. Guo, and D. Wang (2012). Resistance evaluation of 35 potato varieties to the dominant pathogens of potato Fusarium dry rot. Acta Phytophylacica Sinica. 39(4): 308-314.

Chini, A., S. Fonseca, G. Fernández, B. Adie, J.M. Chico, O. Lorenzo, G. Garcíacasado, I. Lópezvidriero, F.M. Lozano, and M.R. Ponce (2007). The JAZ family of repressors is the missing link in jasmonate signalling. Nature. 448(7154): 666671.

Chini, A. (2009). The ZIM domain mediates homo- and heteromeric interactions between Arabidopsis JAZ proteins. Plant J. for Cell and Molecular Biology. 59(1): 77-87.

Du, M., X. Ren, Q. Sun, Y. Wang, and R. Zhang (2012). Characterization of Fusarium spp. Causing Potato Dry Rot in China and Susceptibility Evaluation of Chinese Potato Germplasm to the Pathogen. Potato Research. 55(2): 175-184.

Erayman, M., M. Turktas, G. Akdogan, T. Gurkok, B. Inal, E. Ishakoglu, E. Ilhan, and T. Unver (2015). Transcriptome analysis of wheat inoculated with Fusarium graminearum. Frontiers in Plant Science. 6: 867.

Esfahani, M.N. (2005). Susceptibility assessment of potato cultivars to Fusarium dry rot species. Potato Research. 48(3-4): 215-226.

Florea, L., L. Song, and S.L. Salzberg (2013). Thousands of exon skipping events differentiate among splicing patterns in sixteen human tissues. F1000research. 2: 188.

Frey, M.E., S. D'ippolito, A. Pepe, G.R. Daleo, and M.G. Guevara (2018). Transgenic expression of plantspecific insert of potato aspartic proteases (StAP-PSI) confers enhanced resistance to Botrytis cinerea in Arabidopsis thaliana. Phytochemistry. 149: 1-11.

Fu, Y., L. Shi, M. Sun, W. Shan, X. Wang, and F. Li (2016). Activities of Antioxidative and Cell Wall Defense Enzymes and Expression of StLTPa1 Gene in Fusarium culmorum-infected Potato Tuber. Chinese J. Biological Control. 32(3): 388-395.

Godoy, A.V., A.S. Lazzaro, C.A. Casalongué, and B. San Segundo (2000). Expression of a Solanum tuberosum cyclophilin gene is regulated by fungal infection and abiotic stress conditions. Plant Science. 152(2): 123-134.

Godoy, A.V., M.E. Zanetti, S.B. San, and C.A. Casalongué (2001). Identification of a putative Solanum tuberosum transcriptional coactivator up-regulated in potato tubers by Fusarium solani f. sp. eumartii infection and wounding. Physiol Plant. 112(2): 217-222.

Guevara, M.G., C.R. Oliva, M. Huarte, and G.R. Daleo (2002). An aspartic protease with antimicrobial activity is induced after infection and wounding in intercellular fluids of potato tubers. European J. Plant Pathology. 108(2): 131-137.

Hanin, A.N., G.K.A. Parveez, O.A. Rasid, and M.Y.A. Masani (2020). Biolistic-mediated oil palm transformation with alfalfa glucanase (AGLU1) and rice chitinase $(\mathrm{RCH} 10)$ genes for increasing oil palm resistance towards Ganoderma boninense. Industrial Crops and Products. 144: 112008.

Kidd, P.S., M. Llugany, C. Poschenrieder, B. Gunsé, and J. Barceló (2001). The role of root exudates in aluminium resistance and silicon - induced amelioration of aluminium toxicity in three varieties of maize (Zea mays L.). J. Experimental Botany. 52(359): 1339-1352.

Leach, S.S., and R.E. Webb (1981). Resistance of Selected Potato Cultivars and Clones to Fusarium Dry Rot. Phytopathology. 71(6): 623629.

Leng, N., J.A. Dawson, J.A. Thomson, V. Ruotti, A.I. Rissman, B.M.G. Smits, J.D. Haag, M.N. Gould, R.M. Stewart, and C. Kendziorski (2013). EBSeq: an empirical Bayes hierarchical model for inference in RNA-seq experiments. Bioinformatics. 29(8): 1035-1043.

Lorenzo, O., J.M. Chico, J.J. Sánchezserrano, and R. Solano (2004). JASMONATE-INSENSITIVE1 encodes a MYC transcription factor essential to discriminate between different jasmonateregulated defense responses in Arabidopsis. Plant Cell. 16(7): 1938-1950.

Min, F.X., X.D. Wang, L.S. Hu, Q. Wei, D.X. Zhi, and F.L. Li (2010). Identification of species and pathogenicity of the Fusarium on potato in Heilongjiang Province. Plant Protection. 36(4): 112-115.

Noman, A., A. Hussain, M. Adnan, M.I. Khan, M. Furqanashraf, M. Zainab, K. Alikhan, H.A. Ghramh, and S. He (2019). A novel MYB transcription factor CaPHL8 provide clues about evolution of pepper immunity againstsoil borne pathogen. Microbial Pathogenesis. 137: 103758.

Peters, R.D., A.V. Sturz, M.R. Carter, and J.B. Sanderson (2004). Influence of crop rotation and conservation tillage practices on the severity of soil-borne potato diseases in temperate humid agriculture. Canadian J. Soil Science. 84(4): 397-402.

Radtke, W., and A. Escande (1973). Pathogenicity of cepas from the Fusarium collection on Solanum 
tuberosum cultivars. Rev. Fae. Agron. Balcarce Argent. 49: 62-70.

Sasaki, Y., E. Asamizu, D. Shibata, Y. Nakamura, T. Kaneko, K. Awai, M. Amagai, C. Kuwata, T. Tsugane, T. Masuda, H. Shimada, K. Takamiya, H. Ohta, and S. Tabata (2001). Monitoring of methyl jasmonate-responsive genes in Arabidopsis by cDNA macroarray: selfactivation of jasmonic acid biosynthesis and crosstalk with other phytohormone signaling pathways. DNA Research An International J. for Rapid Publication of Reports on Genes and Genomes. 8(4): 153-161.

Seo, S., M. Okamoto, T. Iwai, M. Iwano, K. Fukui, A. Isogai, N. Nakajima, and Y. Ohashi (2000). Reduced levels of chloroplast FtsH protein in tobacco mosaic virus-infected tobacco leaves accelerate the hypersensitive reaction. Plant Cell. 12(6): 917-932.

Shan, W., Y. Xu, M. Sun, C. Ying, F. Su, X. Sun, X. Li, L. Lei, and F. Li (2017). Evaluation on Disease Resistance of Main Potato Varieties Against $F$. avenaceum and $F$. sporotrichioides Infection in Heilongjiang Province. Crops. 2: 38-43.

Stevenson, W.R., R. Loria, G.D. Franc, and D. Weingartner (2001). Compendium of potato diseases. American Phytopathological Society Press; St. Paul, MN,USA. $100 \mathrm{p}$

Sun, C., X. Zhou, R. Chen, Y. Fan, and L. Wang (2014). Comprehensive Overview of JAZ Proteins in Plants. BIOTECHNOLOGY BULLETIN. 6: 1-
8.

Taif, S., Q. Zhao, L. Pu, X. Li, D. Liu, and X. Cui (2020). A $\beta$-1,3-glucanase gene from Panax notoginseng confers resistance in tobacco to Fusarium solani. Industrial Crops and Products. 143: 111947.

Tornero, P., V. Conejero, and P. Vera (1996). Primary Structure and Expression of a Pathogen-Induced Protease (PR-P69) in Tomato Plants: Similarity of Functional Domains to Subtilisin-Like Endoproteases. Proc Natl Acad Sci U S A. 93(13): 6332-6337.

Uji, Y., S. Taniguchi, D. Tamaoki, and H. Shishido (2016). Overexpression of OsMYC2 Results in the Up-Regulation of Early JA-Rresponsive Genes and Bacterial Blight Resistance in Rice. Plant and Cell Physiology. 57(9): 101.

Wang, J.H., K.D. Gu, P.L. Han, J.Q. Yu, C.K. Wang, Q.Y. Zhang, C.X. You, D.G. Hu, and Y.J. Hao (2020). Apple ethylene response factor MdERF11 confers resistance to fungal pathogen Botryosphaeria dothidea. Plant Science. 291: 110351.

Yang, Z.M., Y. Bi, Y.C. Li, Z.H. Kou, G. Bao, C.K. Liu, Y. Wang, and D. Wang (2012). Changes of Cell Wall Degrading Enzymes in Potato Tuber Tissue Slices Infected by Fusarium sulphureum. Scientia Agricultura Sinica. 45(1): 127-134.

Ye, Q., and G. Wang (1995). On Fusarium Dry Rot of Potato in Zhejiang. Acta Phytopathologica Sinica. 25(2): 148. 